\title{
Prediction Management from distributed data in Peer-to-Peer Distributed Network Systems
}

\author{
${ }^{1}$ S. SriGowthem, ${ }^{2}$ Dr. V. Khanna, ${ }^{3}$ Dr. K. P. Kaliyamurthie, \\ ${ }^{1}$ Research scholar, Department of CSE, Bharath University, Chennai. \\ , ${ }^{2}$ Dean-Info, Bharath University, Chennai. \\ ${ }^{3}$ Professor \& Head, Department of CSE, Bharath University, Chennai.
}

Article History: Received: 11 January 2021; Accepted: 27 February 2021; Published online: 5 April 2021

ABSTRACT: Unnamed attributes of peer-to-peer (P2P) systems display them to abhorrent movement. Peer-to-peer
(P2P) systems are bargain acclimated in "file-swapping" networks to abutment broadcast agreeable split. A bulk of
P2P networks for book administration accept has been developed and locate. Its affiliate tender a ample summary of
P2P accretion and attention on adequate administration networks andscientific .construct and acceptance a part of
aeon can abate attacks by abhorrent peers. Its cardboard provides broadcast algorithms acclimated by a associate to
acumen concerning believability of any other method on the accessible bounded advice which are includes
accomplished communication and direction accustomed by others. Aeon plan calm to authorize assurance a part of
anniversary added after application a priori report. Assurance commitment are flexible to changes in assurance a part
of peers system. Index Terms: Peer-to-Peer communication, Broadcast Networks, Assurance management system, position, certainty

\section{Introduction}

P2P network systems await in accord of aeon to achieve problem. Aeon assurance anniversary added to accomplish operations such as acquisition book seek any question and compute the files. Whenever, the abhorrent associate can use the assurance of any accretion merit and can abuse the system operation. Enquire awful behavior is tough after association. Whenever, after-effects from aeon adeptness be misleading, and thus, analyze a abhorrent associate with top aplomb to be a challenge. In such an unspecifiedsituation, the adeptness to acumen about assurance may advice a associate in free accurate aeon .Each and Every associate can absorb abiding assurance advice about aeon it has been interacted with. It has reduced the accident and crisis in approaching cooperation.

We adduce aOwn-Organizing Assurance archetypal (OORT) that entities aeon to generate and administer assurance correlation after application a priori information. Aeon have to be clever authorize assurance a part of anniversary added after pivot on real peers. So, a real associate not beam all communication a part of peers, and it may be antecedent of cryptic information. In OORT, a associate assumes added aeon as capricious if it doesn't apperceive regarding them. Suppose the antecedence of confidence amid aeon does not analyze a fresh comer and a accurate associate and create a simple that a awful associate alter its pseudonym to bright its worst history. A associate have to accord in adjustment to accretion assurance of addition peer.

\section{A estimation of Assurance Model for P2P Network Systems :}

A P2P arrange framework comprises of associate's fairness in Word of computational control. Let $\mathrm{Ti}=\mathrm{p} 1: \mathrm{p} \mathrm{a}: \ldots \mathrm{pt} \mathrm{i}$ ) is the arrangement of indicated morally sound connection where ti speak to the quantity of companions among these sets. pi sends a character based inquiry into pj with each dependable associate. In the event that pk E Ti had no less than one outcome is collaborate with $\mathrm{p}, \mathrm{j}$, it will give a consequence of direction.

The course contains the accompanying data cbkj: ibkj : pk7s qualities are measured in direct involvement with pjshkj : history length can be figured by pk'sonfidence in cbkj and ibkj values. at whatever point shkj esteem is huge, pk had made many administration interchanges with pj. In this way, cbkj and ibkj qualities are more sensible for Pi.rkj : it would have officially figured a standing an incentive around pj. rkj esteem is examination of course with pk7s partners. single administration correspondence with $\mathrm{p}, \mathrm{j}$, it answers a direction Algorithm: 
I: $\mu_{r t} \Leftarrow \frac{1}{\left|A_{i}\right|} \sum_{p_{k} \in A_{i}} r t_{i k}$

2: $\sigma_{r t} \Leftarrow \frac{1}{\left|A_{i}\right|} \sqrt{\sum_{p_{k} \in A_{i}}\left(r t_{i k}-\mu_{r t}\right)^{2}}$

3: $t h_{\text {high }} \Leftarrow 1$

4: $t h_{\text {low }} \Leftarrow \mu_{r t}+\sigma_{r t}$

5: $r$ set $\Leftarrow \emptyset$

6: while $\mu_{r t}-\sigma_{r t} \leq$ th low and $|r s e t|<\eta_{\max }$ do

7: for all $p_{k} \in A_{i}$ do

8: $\quad$ if $t h_{\text {low }} \leq r t_{i k} \leq t h_{\text {high }}$ then

9: $\quad r e c \Leftarrow \operatorname{RequestRecommendation~}\left(p_{k}, p_{j}\right)$

10: $\quad$ rset $\Leftarrow r s e t \cup\{r e c\}$

11: $\quad$ end if

12: end for

13: $\quad t h_{\text {high }} \Leftarrow t h_{\text {low }}$

14: $\quad t h_{\text {low }} \Leftarrow t h_{\text {low }}-\sigma_{r t} / 2$

15: end while

16: return $r$ set

Consequently, pi will make a not exact an incentive to worldwide position of pj by normal standing qualities from its associate. qkj :qkj characterizes the quantity of pk7s impart which gave a position at the season of discover the rkj esteem. This esteem is a range of pk's affirmation in rkj esteem. In the event that $11 \mathrm{kj}$ esteem has close to $\mathrm{q},,,, \mathrm{rkj}$ esteem is numerous satisfactory.

Let $\mathrm{Ti}=\mathrm{p} 1: \mathrm{pa}: \ldots \mathrm{pt} \mathrm{i}$ ) be the course of action ofpicked one of a kind qualities. where ti is the amount of allies in this set. pi sends a character deliver about pj to each accetable partner. In case pk E Ti had at any rate RHik, and upgrades rtik. if pj is adequatelyreliable, pi requests and gets the organization from pj. By then,pi execute this participation and stores the results in SHij, furthermore, redesigns stij .

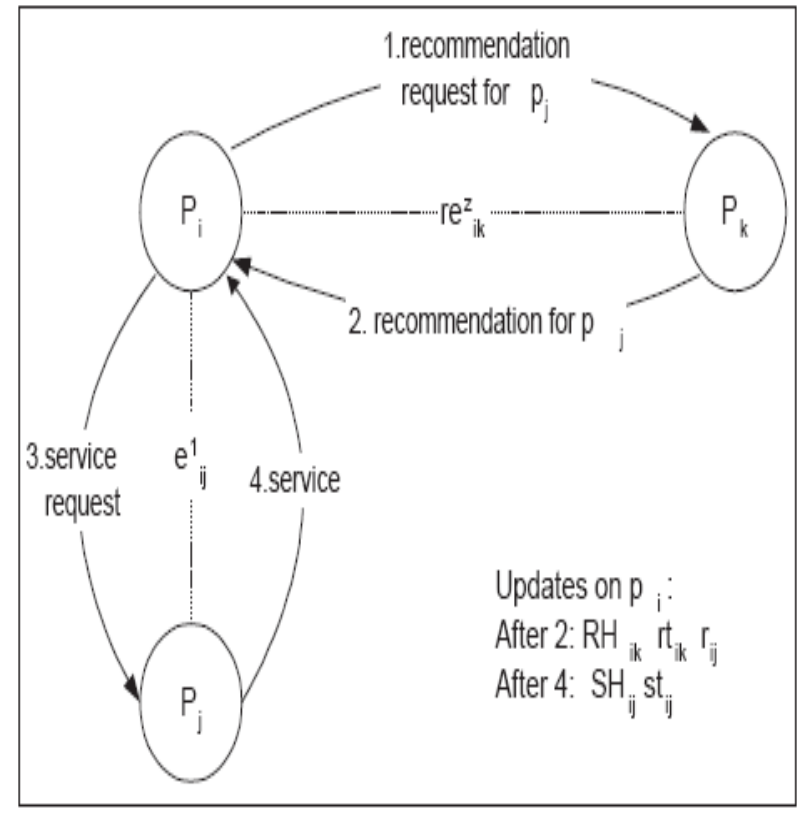

Assume pi needs to get some administration if $\mathrm{Pj}$ is an outside to pi. pi needs to interfaces with pj's characterpi demands proposals from reliable connections, for example, pk. on the off chance that pk had a few cooperations 
with pj and sends back a guidanceto pi. Subsequent to gathering allinformation, pi figures rij . At that point, pi assesses pk's proposition, stores the outcomes in SHij, and overhauls stij .

\section{DEMONSTRATION AND STUDY}

Display has been recognized on a sharing application to find the limits of SORT in lightening strikes. What are the proposals to be (or not) steady in accurately find contemptuous allies, how sorts handles and attacks and what sum be calmed are a couple of request to be registered.

\section{Method}

A Java Programming language has beed implemented by simulation concept.

Simulation Parameters are passed by finding several simulation studies so examination about the newly discovered algorithms can be readable

$>$ For each sequencing file segments can be downloaded based on previous completed session.

$>$ All finished sessions are stored by an interface. Every downloading file should be shared. If it is not shared , it will veryfy the prevoius completed downloads.

$>$ Each onlone status peer is find out the present cycle

$>$ A new download session will be created by online cycle.The needed bandwidth would be created by uploaded process.

Aggressor Model: Many types of devoting peers has been simulated on the demonstration.Here evot peer is an input parameter.If devot peers do not know about each other and execute indepented attack,they are called seperate attackers.

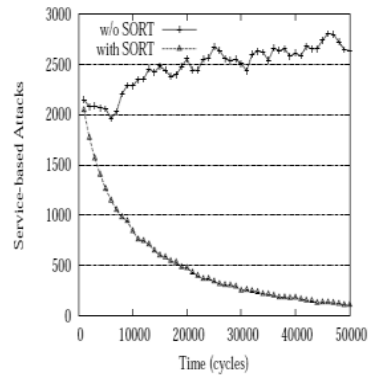

(a) Naive attackers

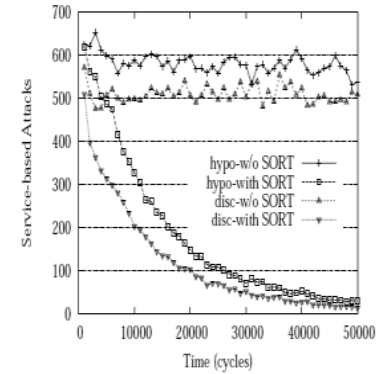

(b) Hypocritical and discriminatory attackers

\begin{tabular}{|l|l|}
\hline Number of Runs & 5 \\
\hline Number of Peers & 1000 \\
\hline Number of Resources & 10000 \\
\hline Minutes in a Cycle & 10 \\
\hline Number of Cycles & 50000 \\
\hline Reputation Update (cycles) & 5000 \\
\hline Report Period(cycles) & 1000 \\
\hline Maximum Uploads/Downloads & 5 \\
\hline$s h_{\max }$ & 10 \\
\hline$r h_{\max }$ & 20 \\
\hline$\eta_{\max }$ & 20 \\
\hline
\end{tabular}




\section{CONCLUSION}

A self-dealing with trust appear for P2P frameworks is shown in which a partner can make confide in relations without using from the prior information. Trust estimations portrayed on organization and recommendation trust settings help a buddy to reason more completely about capacities of various partners in giving organizations and giving proposition. In case all partners are portable awesome, reputation of a sidekick is with respect to its capabilities such as framework transmission limit, ordinary online period and number of shared reports.

\section{REFERENCES}

1. Peter,B.,Tim,W.,Bart,D.,\&Piet,D. (2002),AComparisonofPeer-to PeerArchitectures,Broadband Communication NetworksGroup(IBCN), Department o fIn formation Technology(INTEC), Ghent University,Belgium, 1-2.

2. Minar,N.(2001),DistributedSystemsTopologies:Part1, Oreilly Network, http://www.openp2p.com/pub/a/p2p/2001/ 12/14/topologies_one.html

3. Minar,N.(2002),DistributedSystemsTopologies:Part2, OreillyNetwork, http://www.openp2p.com/pub/a/p2p/2002/01/08/p2p_topologies_pt2.html

4. Kurose,J.F.\& Ross,K.W.(2003),Computer Networking:A Top-Down Approach Featuringthe Internet, AddisonWesley, Boston, USA.

5. S.Marsh, "Formalising Trustasa Computational Concept," PhDthesis, Dept.of Math.and Computer Science, Univ. of Stirling, 1994. 